\title{
Application of Automated complex Gas Reaction Controller for Hydrogen Storage Materials Investigation
}

\author{
Viktor N. Kudiiarov ${ }^{1, a}$, Luydmila V. Gulidova ${ }^{1, b}$, Natalia S. Pushilina ${ }^{1, c}$, \\ Andrey M. Lider ${ }^{1, d}$ \\ ${ }^{1}$ Department of General Physics, Institute of Physics and Technology, National Research Tomsk
Polytechnic University, 30 Lenin Avenue, Tomsk, Russia, 634050

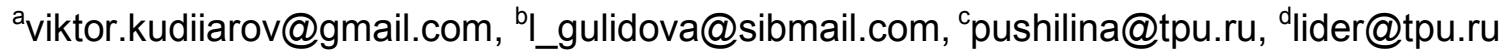

Keywords: Hydrogen storage, Sievert apparatus, Carbon nanotubes.

\begin{abstract}
Automated complex Gas Reaction Controller and its application for hydrogen storage materials investigation are described in this article. Pressure-composition isotherms (PCI) and kinetics measurements of $\mathrm{LaNi}_{5}$ have been obtained for validation of the apparatus. Measurements of both absorption and desorption PCI curves and kinetics measurements at different conditions have been obtained for carbon nanotubes to estimate its hydrogen storage properties.
\end{abstract}

\section{Introduction}

As a result of energy resources depletion and problems of environmental pollution hydrogen has been recognized as an ideal fuel. As high-performance and ecological energy carrier it has big potential for wide application in power engineering, particularly as fuel for transports. One of the general problems is the lack of effective methods of hydrogen storage and transportation. At present different methods of hydrogen storage (under high pressure, in adsorbed state at the cryogenic temperature, in liquid state, in the form of metal hydrides) are investigated [1]. Recently carbon materials, in particular, carbon nanotubes, have been regarded as reservoirs for hydrogen storage [2,3]. In the recent decade these materials have been very popular and different laboratories have published experimental results of sorption capacity. Studies of all peculiarities of hydrogen sorption and desorption by carbon nanotubes are important for science.

The most common method for studying hydrogen sorption by materials is Sievert method [4,5], in which the hydrogen saturation of the samples comes from gaseous atmosphere. Design and key components of the Sievert apparatus automated complex Gas Reaction Controller by Advanced Materials Corporation [6] and methodology for conducting experiments to study the hydrogen sorption and desorption processes have been described in this article. The usability of the complex and the reliability of results have been checked on the $\mathrm{LaNi}_{5}$ samples. Measurements of absorption and desorption PCI curves under different conditions have been obtained for carbon nanotubes to investigate their hydrogen storage properties.

\section{Automated complex Gas Reaction Controller}

Automated complex Gas Reaction Controller, shown schematically in Figure 1, consists of computer (1) software based on LabVIEW, a high-temperature furnace or cryostat (2), the sample (3) chambers of low (4) and high (5) pressure and a controller (6). The controller consists of the electronic control system (7) and a vacuum part. The vacuum part consists of a control volume (8), a low-pressure reservoir (9), high-pressure reservoir (10), pneumatically controlled valves and manual valves, needle valves, pressure gauges, flow control valves and filters. System of emergency hydrogen removal (11), through which hydrogen is removed when exceeding the maximum allowable pressure, have been installed for security purposes between reservoirs (low and high pressure). Vacuum (12) assembly, consisting of a forevacuum membrane pump and turbomolecular pump, is used for creation the vacuum in the vacuum system. Hydrogen is supplied into the vacuum system from the cylinder (13) or generator (14). Cooling system (15) is installed between the furnace and the controller for prevention of damage of connecting wires from the heating. 


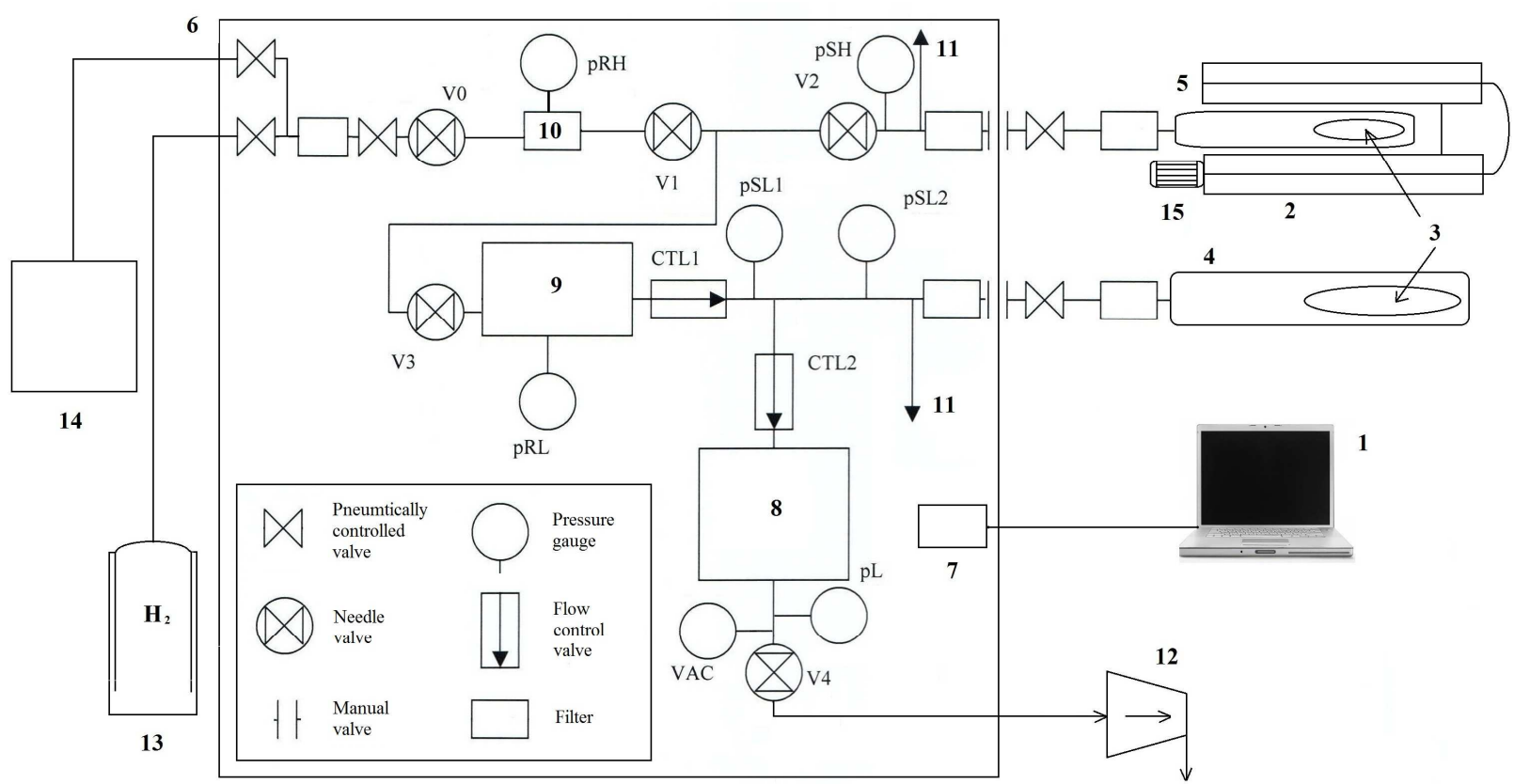

Fig. 1. The scheme of the automated complex Gas Reaction Controller: 1 - computer; 2 furnace/cryostat; 3 -sample; 4 - low-pressure chamber; 5 - high-pressure chamber; 6 - controller; 7

- electronic control system; 8 - control volume; 9 - low-pressure reservoir; 10 - high-pressure reservoir; 11 - system of emergency hydrogen removal; 12 - vacuum assembly; 13 - cylinder with hydrogen; 14 - hydrogen generator; 15 - cooling system

The using the cryostat and high-temperature furnace allows conducting studying in the temperature range from $-30{ }^{\circ} \mathrm{C}$ to $1000^{\circ} \mathrm{C}$. Linear heating of the sample in the chamber at a given rate of heating at a constant pumping are used when working with high temperatures. Constant pumping is necessary to remove desorbed gas during heating. Linear heating at a given rate and the possibility of maintain a high vacuum in the chamber makes it possible to conduct vacuum annealing of samples. Maximum sample size is limited by the chambers sizes: $\varnothing 30 \mathrm{~mm}$ to $80 \mathrm{~mm}$ for low-pressure chamber and $\varnothing 6 \mathrm{~mm}$ to $20 \mathrm{~mm}$ for high-pressure chamber. Maximum pressure of hydrogen in the low-pressure chamber is $202.64 \mathrm{kPa}$, in the high-pressure chamber is $5066 \mathrm{kPa}$.

There is one main method of hydrogen storage materials investigation by automated complex Gas Reaction Controller - PCI curves fitting. PCI (pressure-composition isotherm) is the dependence of the maximum amount absorbed (PCI absorption) or desorbed (PCI desorption) hydrogen on pressure in the chamber at a constant temperature. By using PCI method it is possible to obtain important information about the nature of hydrogen absorption and desorption reactions, such as hysteresis of hydrogen sorption and desorption and its kinetics.

\section{Experiments and results}

Experiments on the hydrogen sorption and desorption processes investigation run in the following order: the sample is weighed, the weight and density are entered into the complex software, the sample is placed into the chamber, the chamber is connected to the vacuum system and evacuated, and the sample is heated or cooled with continuous pumping. Then PCI method is applied.

Pressure-composition isotherms (PCI) and kinetics measurements of $\mathrm{LaNi}_{5}$ have been obtained for validation of the apparatus. $\mathrm{LaNi}_{5}$ sample wrapped in quartz wool to prevent chamber from small metal particles formed during hydrogen sorption and desorption processes was placed in the high-pressure chamber. The sample was activated for hydrogen sorption by hydrogen pumping in and pumping out several times. Then the study was conducted by the PCI method at high pressures and room temperature. Figure 2 shows PCI curves for absorption $(\mathrm{a}, 1)$ and for desorption $(\mathrm{a}, 2)$ and hydrogen absorption kinetics (b) of $\mathrm{LaNi}_{5}$. 

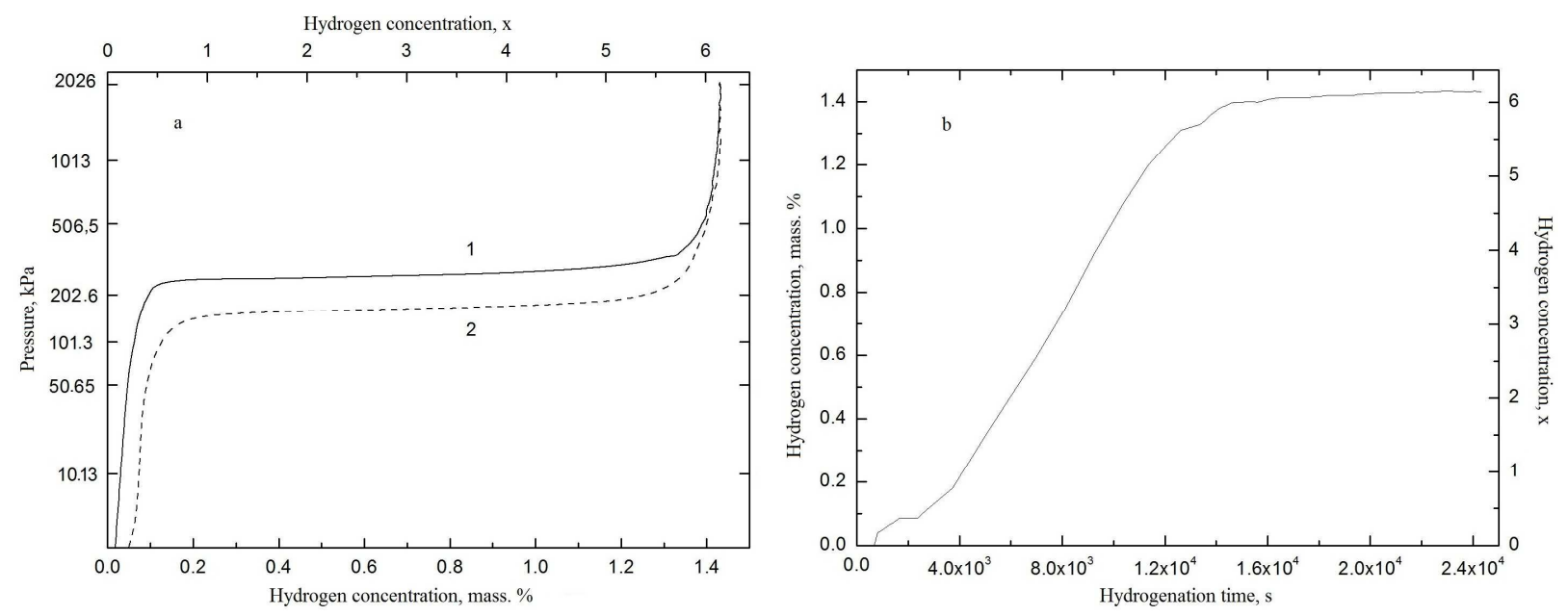

Fig. 2. PCI curves for absorption (a,1) and for desorption (a,2) and absorption kinetics (b) of $\mathrm{LaNi}_{5}$

$\mathrm{LaNi}_{5}$ is a very well studied hydrogen storage material and can absorb large amounts of hydrogen at room temperature to form a hydride $\mathrm{LaNi}_{5} \mathrm{H}_{6}$ and it used for the Sievert apparatus calibration $[5,6]$. The results presented in Figure 2 are in good agreement with the results of other authors $[5,6]$. This indicates that the complex is properly designed and precisely assembled and results obtained by using the complex - are reliable.

Measurements of absorption and desorption PCI curves and kinetics measurements of carbon nanotubes have been done under the following conditions: the pressure range was from 0 atm to 8 atm, room temperature and low-temperature $\left(-30^{\circ} \mathrm{C}\right)$. PCI curves at the room (a) and low (b) temperature are shown in Figure 3. Figure 4 shows the hydrogen sorption (1) and desorption (2) kinetics at the room (a) and low temperature (b).

The results show that the amount of hydrogen adsorbed by samples at the low temperature is in five times more than at the room temperature. The maximum hydrogen sorption capacity of carbon nanotubes at low temperature is about $4.4 \mathrm{wt} \%$. The higher pressure of experiment is needed for increasing the amount of absorbed hydrogen and finding the limit of hydrogen saturation. Also the absolute hydrogen desorption at the low temperature is observed. According to USA Department of Energy (DOE) the minimum hydrogen sorption capacity of porous materials with high surface area must be about $6.5 \mathrm{wt} \%$ for effective using it as hydrogen storage [1].
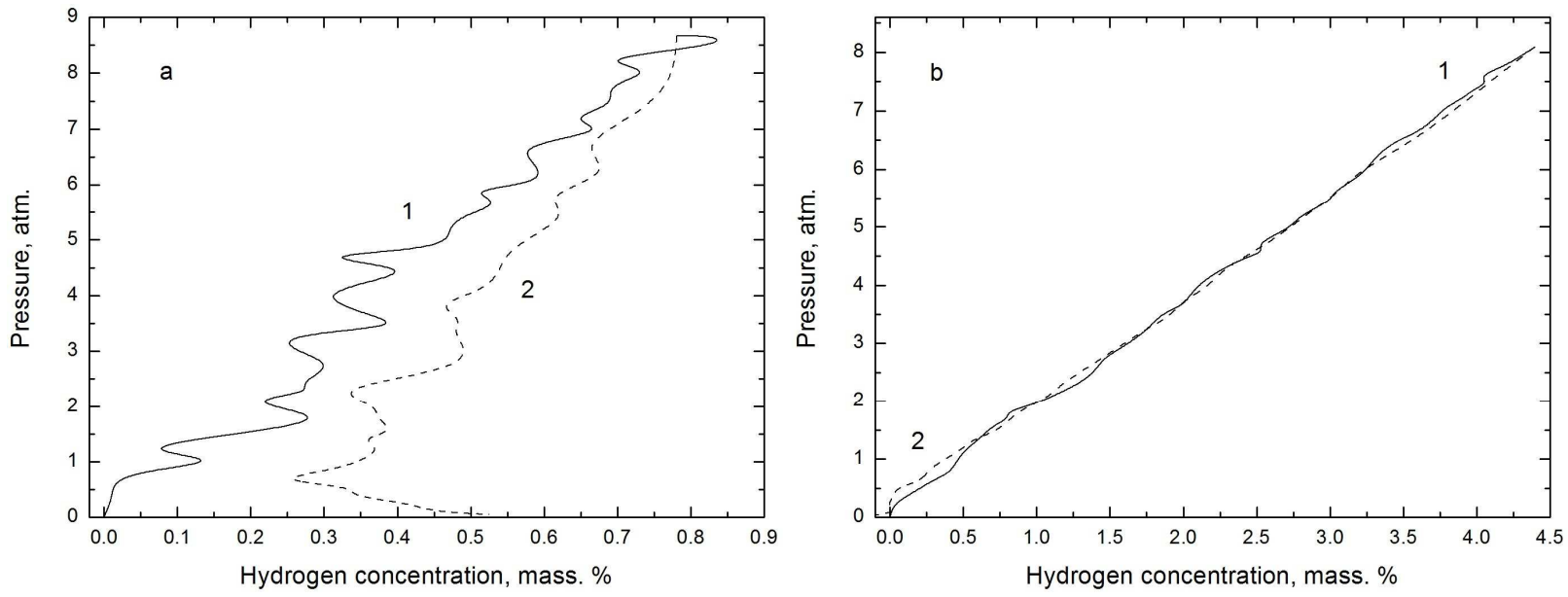

Fig. 3. PCI curves for hydrogen absorption (1) and for desorption (2) of carbon nanotubes at the room (a) and low temperature (b) 

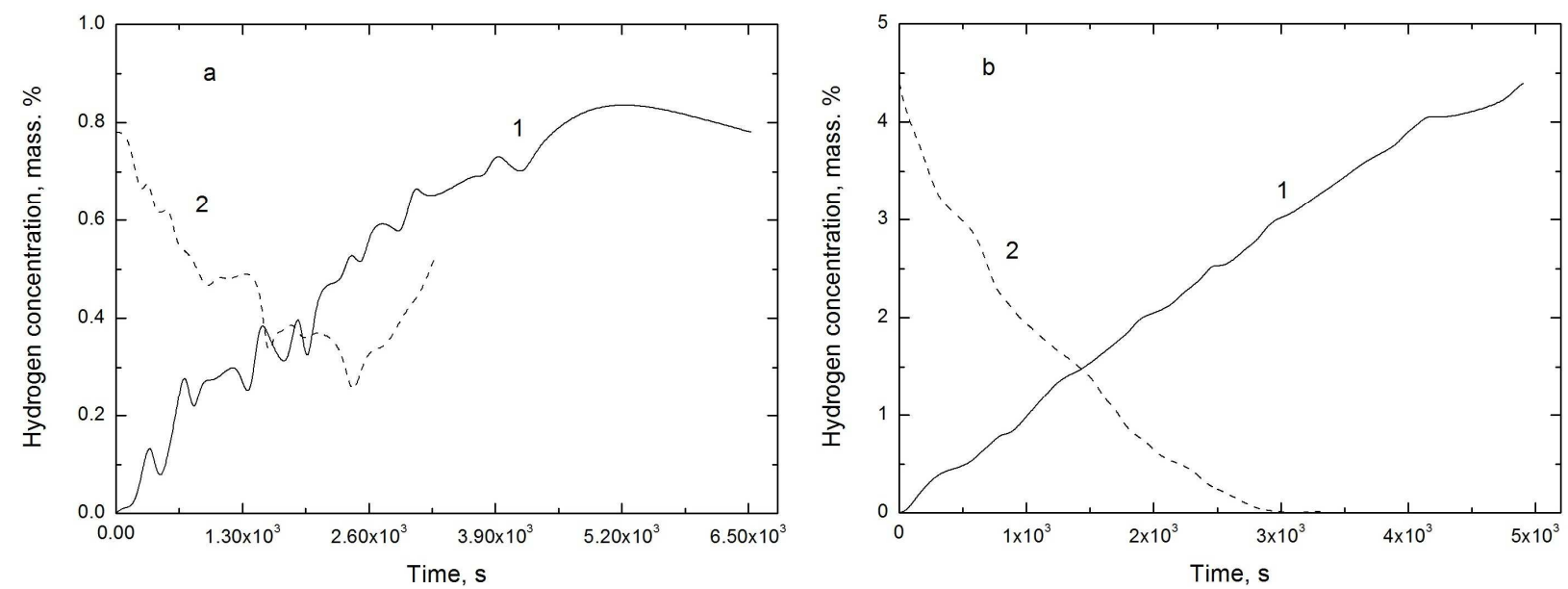

Fig. 4. Kinetics of hydrogen absorption (1) and desorption (2) of carbon nanotubes at the room (a) and low temperature (b)

With increasing the time the amount of absorbed hydrogen by sample increases regardless of the process temperature. The time of hydrogen desorption from the samples is longer than sorption time. The partial hydrogen desorption of carbon material at room temperature is observed. At low temperature this effect was not detected.

\section{Summary}

Design and key components of the automated complex Gas Reaction Controller and methodology for conducting experiments to study the hydrogen sorption and desorption processes have been described. The usability of the complex and the reliability of results have been checked on the $\mathrm{LaNi}_{5}$ samples.

Investigation of hydrogen storage by carbon nanotubes showed that the amount of hydrogen adsorbed by samples at the low temperature is in five times more than at the room temperature. The maximum hydrogen sorption capacity by carbon nanotubes at low temperature is about $4.4 \mathrm{wt} \%$. The higher pressure for experiment is needed for increasing the amount of absorbed hydrogen and finding the limit of hydrogen saturation. At the room temperature incomplete hydrogen desorption is observed while this effect has not been detected for low temperature.

\section{References}

[1] G.E. Ioannatos, X.E. Verykios, $\mathrm{H}_{2}$ storage on single- and multi-walled carbon nanotubes, International Journal of Hydrogen Energy. 35 (2010) 622-628.

[2] S.Y. Lee, S.J. Park, Effect of temperature on activated carbon nanotubes for hydrogen storage behaviors, International Journal of Hydrogen Energy. 35 (2010) 6757-6762.

[3] N. Nishimiya, K. Ishigaki, H. Takikawa, M. Ikeda, Y. Hibi, T. Sakakibara, A. Matsumoto, K. Tsutsumi, Hydrogen sorption by single-walled carbon nanotubes prepared by a torch arc method, Journal of Alloys and Compounds. 339 (2002) 275-282.

[4] T. Voskuilen, Y. Zheng, T. Pourpoint, Development of a Sievert apparatus for characterization of high pressure hydrogen sorption materials, International Journal of Hydrogen Energy. 35 (2010) 10387-10395.

[5] H.H. Cheng, X.X. Deng, S.L. Li, W. Chen, D.M. Chen, K. Yang, Design of PC based high pressure hydrogen absorption/desorption apparatus, International Journal of Hydrogen Energy. 32 (2007) 3046-3053.

[6] http://www.advanced-material.com/ 